\title{
Estimation of Normal Biochemical Parameters in Indigenous Pig of Assam: A Preliminary Study
}

\author{
Jumi Dutta $^{1 *}$, Moffajel Hussain ${ }^{2}$, Santosh Upadhyaya ${ }^{1}$ and K. Pame ${ }^{3}$ \\ ${ }^{1}$ Department of Veterinary Biochemistry, College of Veterinary Science, \\ Khanapara, Guwahati, India \\ ${ }^{2}$ Department of Chemistry, Royal Global University, Guwahati, India \\ ${ }^{3}$ Department of Livestock Products and Technology, LCVSc, Joyhing, Lakhimpur, India \\ *Corresponding author
}

\section{A B S T R A C T}

\section{Keywords}

ALT, AST, Blood profile, Mineral profile, Susscrofa scrofa

Article Info

Accepted:

07 August 2020

Available Online:

10 September 2020
The blood of pig (Susscrofa scrofa) was taken for the experiments to find out the normal biochemical parameters. The blood was collected from five randomly selected animals (Pig) irrespective of gender in AICRP pig of C. V. Sc. Khanapara. The blood was collected from the anterior venacava. The plasma was separated by centrifuging at $3000 \mathrm{rpm}$ for $15 \mathrm{~min}$. The blood profile activity was estimated on the day of collection. The level of blood glucose, total protein, cholesterol, creatinine, blood urea nitrogen, liver specific enzymes like AST, ALT along with mineral profile calcium, phosphorus were estimated. It was concluded that the blood parameters were found to be in the normal range.

\section{Introduction}

The domestic pig (Susdomestica) has served for more than 20 years as a model animal for biomedical research on human disease, especially in hematological, cardiac, cardiovascular, immunological, and gastrointestinal studies (Dodds, 1982). The greatest strengths of pigs as animal models are their physiological, anatomical, nutritional, and metabolic similarities to humans (Dodds, 1982). During the last few decades swine production has continued to grow and flourish in both developed and developing countries in order to meet the marked increased demand for pork with increase human population. The trend in swine production is too large, more intensive confinement systems. The number of pork producers in Northeast has been increase by one-third over the past decade and yet the number of pigs marketed per year has decreased by more than one million. The normal biochemical parameters are important in assessing the state and position of disease to various pathological situations. Pigs are 
used more frequently as medical models of human diseases such as dyslipidemia, cardiovascular diseases (atherosclerosis, varicose vein), type 2 diabetes and gastrointestinal diseases (inflammatory bowel syndrome) etc. however, the normal physiological values of biochemical parameters of pigs are not frequently available like rats or mice.

The main objective of the present study was to accumulate the normal reference values for commonly measured biochemical parameters in indigenous pigs of Assam.

\section{Materials and Methods}

\section{Period of Study}

The observation in the present study was made for a period of one and half month from $1^{\text {st }}$ February to $15^{\text {th }}$ March, 2019. A total five random animals (Pig) from AICRP pig of C.V. Sc. Khanapara, were selected for the experiment.

\section{Collection of Blood}

Blood samples were collected from five random animals (pig). The experimental animals were restrained in ventro-dorsal position \& blood samples were collected directly from anterior venacava under aseptic condition by using 15 gauge four 4 inches needles and transferred to $15 \mathrm{ml}$ glass centrifuge tube. The blood was allowed to clot in room temperature. Then the tube was centrifuge at $3000 \mathrm{rpm}$ for 15 minute. The serum was separated kept in plastic vials and stored in deep freeze at $-20^{\circ} \mathrm{c}$ for estimation of blood biochemical constituent.

\section{Biochemical Analysis}

Blood biochemical constituent viz. Blood Glucose, Total Protein, Blood Urea Nitrogen,
Creatinine, SGPT, SGOT, Cholesterol, Calcium \& Phosphorus were estimated by available kit procured from Merck Pvt. Ltd.

\section{Results and Discussion}

The differences in the values of the biochemical parameters are observed due to variation in age, sex, breed or strain, sampling technique and testing methodology.

Blood glucose appears to be one of the key nutrients affecting cyclicity in farm animals and a minimum level of $40-60 \mathrm{mg} / \mathrm{ml}$ is required to maintain the physiological processes of the body ${ }^{[8]}$. Low blood glucose may be associated with infertility. In analyses made on various fasting pigs, it seems that the older pigs tend to have higher blood sugar levels. It has been shown by several authors that there is an intimate relationship between the concentration of glucose and inorganic phosphate in the blood ${ }^{[12]}$. The blood sugar in the fasting pigs varied between 40 and $74 \mathrm{mg}$ per cent.

Proteins are the building blocks of all cells and body tissues. They act as transport substances of hormones, vitamins, minerals, lipids and other materials. Thus, protein act as working horses of the cell ${ }^{[14]}$. Plasma protein albumin, fibrinogen, prothrombin, and the gamma globulins, which constitute $6 \%$ to $7 \%$ of the blood plasma. Proteins maintain osmotic pressure, increase blood viscosity, and help to maintain blood pressure. All the plasma proteins except the gamma globulins are synthesized in the liver. Pigs with a higher albumin concentration, a lower gammaglobulin concentration, a higher copper concentration and a higher creatine kinase activity in serum showed a higher daily weight gain ${ }^{[1]}$.

Bloodurea nitrogen concentration in blood may decrease with impaired conversion of 
ammonia to urea by the liver. Low serum urea concentrations are, however, not specific for liver disease. Low urea nitrogen concentration is also seen in anorectic patients consuming less protein. In ruminants that are anorectic or on a low-protein diet, rumen microbes recur to BUN as a nitrogen source for their own protein synthesis, decreasing the BUN concentration (In Veterinary Medicine, Eleventh Edition, 2017). Blood urea nitrogen (BUN) is a serum byproduct of protein metabolism. It is one of the oldest prognostic biomarkers in heart failure. Urea is formed by the liver and carried by the blood to the kidneys for excretion. Diseased or damaged kidneys cause BUN to accumulate in the blood as glomerular filtration rate (GFR) drops. Conditions such as shock, heart failure, a high protein diet, and bleeding into the gastrointestinal tract can cause BUN elevations $^{[17]}$.

Serum creatinine is an important indicator of renal health because it is an easily measured byproduct of muscle metabolism that is excreted unchanged by the kidneys. Creatinine itself is produced via a biological system involving creatine, phosphocreatine (also known as creatine phosphate), and adenosine triphosphate ${ }^{[4]}$. It is synthesized primarily in the liver from the methylation of glycocyamine (guanidino acetate, synthesized in the kidney from the amino acids arginine and glycine) by S-adenosyl methionine. It is then transported through blood to the other organs, muscle, and brain, where, through phosphorylation, it becomes the high-energy compound phosphocreatine ${ }^{[15]}$. Creatine conversion to phosphocreatine is catalyzed by creatine kinase; spontaneous formation of creatinine occurs during the reaction.

Serum calcium has a critical role in the metabolism of the body including the cells of reproductive system ${ }^{[12]}$. Calcium must always be maintained to assure the proper cellular functions of the animal. The ovarian activity of ruminants is influenced by calcium deficiency. Calcium along with phosphorus are important in skeletal structure development, but their presence in soft tissues is also vitally important. Both aid in blood clotting, muscle contraction, and energy metabolism. About 99 percent of the calcium and 80 percent of the phosphorus in the body are found in the skeleton and teeth. Therefore, deficiency of calcium and phosphorus will result in impaired bone mineralization, reduced bone strength, and poor growth.

Young pigs with a deficiency of calcium and phosphorus will have clinical sings of rickets. Mature pigs eating a deficient diet will remove calcium and phosphorus from the bone (osteoporosis), decreasing bone strength. This can result in a condition called "Downer Sows" and can be prevented by proper diet formulation (Swine Nutriton Guide).

A decrease in the serum inorganic phosphate is one of the first manifestations of phosphorus deficiency in pigs. Blood samples obtained from pigs purchased for laboratory use have been found to have a low serum inorganic phosphorus as well as low calcium. Phosphorus (P) is of vital importance for many aspects of metabolism, including bone mineralization, blood buffering, and energy utilization $^{[2]}$.

Phosphorus is mainly combined with calcium and is found in the bones. Approximately $15 \%$ exists as inorganic phosphorus or phosphate esters. It is involved in the carbohydrate metabolism and is a component of many other substances. Increased levels are found in hypoparathyroidism, renal failure, bone metastatis and liver diseases. Decreased levels are found in hyperparathyroidism, rickets and Vitamin D deficiency. 
Table.1 Values of blood parameters of Indigenous pig (Susscrofascrofa)

\begin{tabular}{|c|c|c|c|c|c|c|}
\hline Parameter & $\begin{array}{c}\text { Standard } \\
\text { Value }\end{array}$ & \multicolumn{5}{|c|}{ Sample } \\
\cline { 5 - 7 } & S-1 & S-2 & S-3 & S-4 & S-5 \\
\hline Blood Glucose (mg/dl) & $85-150$ & 113.34 & 95.10 & 130.33 & 120.23 & 117.48 \\
\hline Total protein (gm/dl) & $7-9$ & 6.46 & 7.21 & 7.87 & 9.30 & 6.89 \\
\hline Creatinine (mg/dl) & $1-2.5$ & 0.91 & 1.31 & 2.03 & 2.45 & 1.23 \\
\hline $\begin{array}{c}\text { Blood Urea } \\
\text { Nitrogen(mg/dl) }\end{array}$ & $10-30$ & 15.79 & 31.40 & 20.45 & 19.72 & 23.73 \\
\hline Cholesterol (mg/dl) & $36-54$ & 39.89 & 43.01 & 48.42 & 55.32 & 38.21 \\
\hline ALT (U/L) & $31-59$ & 43.21 & 38.54 & 30.61 & 47.44 & 50.33 \\
\hline AST (U/L) & $32-84$ & 25.71 & 32.11 & 67.43 & 71.82 & 60.33 \\
\hline Calcium (mg/dl) & $7.1-11.6$ & 9.06 & 12.33 & 10.37 & 11.44 & 10.84 \\
\hline Phosphorus (mg/d) & $5.3-9.6$ & 4.32 & 6.98 & 8.31 & 5.21 & 4.33 \\
\hline
\end{tabular}

Cholesterol is the precursor of steroid hormone. Epidemiological studies, clinical trials, and controlled experiments with animals and humans have demonstrated that strong relationships exist between different dietary variables, plasma and lipoprotein cholesterol concentrations, and development of atherosclerosis and cardiovascular disease $^{[10]}$. Cholesterol balance in the body is determined, in part, by the excretion of bile acids and neutral steroids, and steroid excretion is considered one of the most important regulatory mechanisms in cholesterol metabolism. The roles of bile acid and neutral steroid excretion in regulating cholesterol metabolism are discussed by ${ }^{[6]}$. A number of studies have measured steroid excretion in the pig.

Alanine Aminotransferase (ALT), also referred to as glutamate pyruvate transaminase (GPT) is an enzyme involved in amino acid metabolism. It is found in many tissues, but the highest levels are found in liver and kidney tissues. ALT found in the cytoplasm of hepatocytes is also found in mitochondria but generally at considerably lower concentrations depending on species and tissuees. Serum ALT has been recognized as a marker of hepatocellular injuiry since the $1950 \mathrm{~s}^{[5]}$. Numerous studies using carbon tetrachloride have clearly shown the value of serum ALT as an indicator of hepatocellular necrosis, especially in dogs and cats but to a much lesser extent in horses, cattle, swine, sheep, and goats ${ }^{[9,13]}$.

Mild to moderate increase in serum ALT activity are also observe in dogs and cats with endocrine disease such as diabetes mellitus, hyperthyroidism, hyperadrenocorticism, and hypothyroidism. Dogs with diabetes mellitus have increased serum ALT activity ${ }^{[5]}$. Cats with Diabetes ketoacidosis commonly have increase serum Alt activity ${ }^{[3]}$.

Aspartate aminotransferase (AST) also referred to as glutamate oxaloacetate transaminase (GOT), is an enzyme involved in amino acid metabolism. The activity of AST is in the serum is significantly increased during heart, liver, kidney and muscle diseases and tissue injuries. The activity of the enzyme is increased 4-8 hours following a myocardial infarction, reaching its peak in 2-3 days and declining on the fifth and sixth days.

AST activity is relatively high and in similar amounts in liver and in skeletal and cardiac muscle, but it varies between species ${ }^{[4,11]}$. AST is located in the cytosol but is in higher concentrations in mitochondria. There is only 
$48.1 \%$ amino acid sequence homology between cytosolic AST (cAST) and mitochondrial AST (mAST) from horse heart [10].

Increased serum AST activity is observed with both reversible and irreversible injury to hepatocytes and can be seen following hepatocellular injury and cholestasis, similar to serum ALT activity in dogs, swine, cats and goats. The diagnostic sensitivity of serum AST activity in animals has been reported as $72 \%$ for hepatic necrosis and $100 \%$ for hepatic lipidosis ${ }^{[16]}$.

In conclusion the biochemical profile obtained from this study was within the range of the standard value. Biochemical parameters are good indicators of the physiological and pathological changes in the animals. Blood parameters are indispensible in the diagnosis, treatment or prognosis of many diseases. In conclusion, the closeness of the biochemical values obtained from the pigs is an indication that the result may be used as baseline data for pigs reared in Assam. The result is also a demonstration that pigs used in this experiment were apparently healthy animals.

\section{References}

1. A. R. W. Elbers, G. H. M. Counotte\& M. J. M. Tielen. Haematological and clinicochemical blood profiles in slaughter pigs, Veterinary Quarterly. 1992:14:2, 5762

2. Alexander L, Qu A, Cutler S, Mahajan A, Rothschild M, Cai W, Dekkers J, Stahl C. A calcitonin receptor (calcr) single nucleotide polymorphism is associated with growth performance and bone integrity in response to dietary phosphorus deficiency. J Anim Sci. 2010:88: 10091016.

3. Bergmeyer HU, Bowers GN Jr, et al: ClinChem 1977; 23: 887.
4. Boyd, J. W. The mechanisms relating to increases in plasma enzymes and isoenzymes in diseases of animals. Vet. Clin. Path. 1983: 12, 9-24.

5. Coles, E.H. Clinical Enzymolozy. In veterinary Clinical Pathology by Coles, E.H. $4^{\text {th }}$ edn. W. B. Sounders Co. 1986: pp.114-127.

6. Dietschy, J. M., and J. D. Wilson. Regulation of cholesterol metabolism. I. Synthesis. New Engl. J. Med. 1970. 282:1128-1138.

7. Dodds, W. J. The pig model for biomedical research. Fed. Proc. 1982. 41:247-256.

8. Dukes, H.H. The physiology of domestic Animals (c.f. Melvin J. Swenson and William O. Reece Edn.). 1996. P.42, Comstock Publishing Associates. Ithaca and London.

9. Everett, R.M., Duncan, J.R., and Prasse, K.W. Alkaline phosphatase, leucine aminopeptidase, and alanine aminotransferase activities with obstructive and toxic disease in cats. Am.J. Vet. Res. 1977. 38, 963-966.

10. Kaneko, J. J.; Harvey, J. W. and Bruss, H. C. Clinical Biochemistry of Domestic Animals $6^{\text {th }}$ (Edn.) Academic press, New York.1997

11. Keller, P. Emzyme activities in the dog: tissue analysis, plasma values and intercellular distribution. Am. J. Vet. Res. 1981. 42, 575-582.

12. McCullagh, E. P., and McCullagh, D. R., J. Lab. and Clin. Med., 1932: 17, 754.

13. Noonan, N.E. Variation of plasma enzymes in the pony and the dogs after carbon tetrachloride administration. Am. J. Vet. Res. 1981:42, 674-678

14. Satyanarayana, U. Biochemistry. $2^{\text {nd }}$ Edn., Books and Allied (P) Ltd., Chintamoni Das Lane, Kolkata. 2002. P.45.

15. Taylor, E. Howard. Clinical Chemistry. New York: John Wiley and Sons. 1989. pp. 4, 58-62. 
16. West, H. J. Evolution of total plasma bile acid concentrations for the diagnosis of hepatobiliary diseases in horses. Res.

Vet. Sci.1989: 46, 264-270.
17. Y. Xue, Navaid Iqbal. Reference Module in Biomedical Sciences. 2014.

\section{How to cite this article:}

Jumi Dutta, Moffajel Hussain, Santosh Upadhyaya and Pame, K. 2020. Estimation of Normal Biochemical Parameters in Indigenous Pig of Assam: A Preliminary Study. Int.J.Curr.Microbiol.App.Sci. 9(09): 670-675. doi: https://doi.org/10.20546/ijcmas.2020.909.084 